\title{
SHARP BOUNDS FOR THE GREEN'S FUNCTION AND THE HEAT KERNEL
}

\author{
Peter Li, Luen-fai Tam, AND Jiaping Wang
}

\section{$\S 0$ Introduction}

The main purpose of this note is two-fold. The first is to give simple proofs to some recent theorems of Colding-Minicozzi in [C-M] on the asymptotic behavior of the Green's function on manifolds with non-negative Ricci curvature and maximal volume growth. The second is to give sharp upper and lower estimates for the heat kernel on this class of manifolds. In fact, after integrating these estimates with respect to the time variable, they yield sharp pointwise bounds for the Green's function. The interested reader should refer to [C-M] for a detailed history of this subject.

Throughout this paper, we assume that $M^{n}$ is an $n$-dimensional complete noncompact manifold with non-negative Ricci curvature. With the exception of Theorem 2.1 and Corollary 2.3, we will assume that $n \geq 3$. Let us denote the distance between $x, y \in M$ by $d(x, y)$. If $B_{x}(r)$ denotes the geodesic ball of radius $r$ centered at $x$, then we denote $V_{x}(r)$ and $A_{x}(r)$ to be the volume and the area of $B_{x}(r)$ and $\partial B_{x}(r)$, respectively. In all the theorems, with the exception of Theorem 1.2, we will assume that $M$ has maximal volume growth. This means that for some fixed point $p \in M$,

$$
\liminf _{r \rightarrow \infty} r^{-n} V_{p}(r)>0 .
$$

If we define

$$
\theta_{p}(r)=n^{-1} r^{1-n} A_{p}(r),
$$

then the Bishop volume comparison theorem readily $[\mathrm{B}-\mathrm{C}]$ asserts that there exists $\theta>0$ independent of $p$, such that

$$
\theta_{p}(r) \searrow \theta
$$

and

$$
r^{-n} V_{x}(r) \searrow \theta
$$

Received October 30, 1996.

The first author's research was partially supported by NSF grant \#DMS-9626310.

The second author's research was partially supported by an Earmarked grant of Hong Kong. The third author's research was supported by an AMS Centennial Fellowship. 
as $r \rightarrow \infty$. Let $G(x, y)$ be the minimal positive Green's function on $M$, whose existence is guaranteed by $[\mathrm{V}]$ and $[\mathrm{L}-\mathrm{Y}]$. It also follows from the estimates in [L-Y] and (0.2) that there exists a positive constant $C(n, \theta)$ depending only on $n$ and $\theta$ such that

$$
C^{-1}(n, \theta) d^{2-n}(x, y) \leq G(x, y) \leq C(n, \theta) d^{2-n}(x, y) .
$$

Moreover, the Laplacian comparison theorem $[\mathrm{G}-\mathrm{W}]$ implies that, for any $x \in M$,

$$
\Delta_{y} d^{2-n}(x, y) \geq 0
$$

in the sense of distribution. Finally, the gradient estimate in $[\mathrm{Cg}-\mathrm{Y}]$ asserts that there exists a positive $C(n)>0$ depending only on $n$, such that, if $u$ is a positive harmonic function defined on $B_{x}(R)$ then

$$
|\nabla \log u|(x) \leq C(n) R^{-1}
$$

\section{$\S 1$ Asymptotic behavior of the Green's function}

We are now ready to give a simple proof to Theorem 0.1 of $[\mathrm{C}-\mathrm{M}]$.

Theorem 1.1. Let $M^{n}$ be an $n$-dimensional $(n \geq 3)$ Riemannian manifold with non-negative Ricci curvature. If $M$ has maximal volume growth, then the Green's function $G(p, x)$ with a pole at any fixed point $p \in M$ must satisfy the asymptotic behavior

$$
\lim _{x \rightarrow \infty} \frac{n(n-2) \theta G(p, x)}{d^{2-n}(p, x)}=1 .
$$

Proof. Let us denote the cut locus to the point $p \in M$ by $E$, and let $\Omega$ be the complement of $E$ in $M$. Since $E$ is of measure zero, Fubini's theorem asserts that the set $\partial B_{p}(r) \cap E$ has $(n-1)$-dimensional measure zero for almost all $r$. We say such $r$ is permissible. By mollifying the function $d_{p}(\cdot)=d(p, \cdot)$, one obtains a sequence of smooth functions $\left\{f_{k}\right\}$ on $M$ such that $f_{k}$ converges to $d_{p}(x)$ uniformly on compact subsets of $M$. Moreover, $\left|\nabla f_{k}\right| \leq 2$ on $M$ and $\nabla f_{k}$ converges to $\nabla d_{p}$ on compact subsets of $\Omega$. It is also well known that (for example, see $[\mathrm{C}]$ ) it is possible to choose $f_{k}$ so that $\Delta f_{k} \leq \frac{n-1}{d_{p}}+1$ on $M$, and that $\Delta f_{k}$ converges to $\Delta d_{p}$ in the sense of distribution. Let $h_{k}(x)=f_{k}^{2-n}(x)$ and $h(x)=d_{p}(x)^{2-n}$. Then for any permissible $r$, the fact that $\left|\nabla f_{k}\right| \leq 2$ and $\nabla f_{k}$ converges to $\nabla d_{p}$ on $\partial B_{p}(r) \cap \Omega$ implies that

$$
\begin{aligned}
\lim _{k \rightarrow \infty} \int_{B_{p}(r)} \Delta h_{k}(y) d y & =\lim _{k \rightarrow \infty} \int_{\partial B_{p}(r)} \frac{\partial}{\partial \nu}\left(h_{k}(y)\right) d y \\
& =\lim _{k \rightarrow \infty} \int_{\partial B_{p}(r) \cap \Omega} \frac{\partial}{\partial \nu}\left(h_{k}(y)\right) d y \\
& =\int_{\partial B_{p}(r) \cap \Omega} \frac{\partial}{\partial \nu}(h(y)) d y \\
& =-(n-2) r^{1-n} A_{p}(r) \\
& =-n(n-2) \theta_{p}(r) .
\end{aligned}
$$


For permissible $R$ and $R_{0}$ with $1<R_{0}<R$, let us denote $B_{0}=B_{p}\left(R_{0}\right)$. Let $x$ satisfies $d_{p}(x)=r$ with $R_{0} \leq r \leq \frac{R}{2}$, then the divergence theorem implies that

$$
h_{k}(x)=-\int_{B_{p}(R)} G(x, y) \Delta h_{k}(y) d y+\int_{\partial B_{p}(R)}\left(G(x, y) \frac{\partial h_{k}}{\partial \nu_{y}}-h_{k} \frac{\partial}{\partial \nu_{y}} G(x, y)\right),
$$

where $\nu_{y}$ is the unit outward normal of $\partial B_{p}(R)$. This can be rewritten as

$$
\begin{aligned}
h_{k}(x)+ & G(p, x) \int_{B_{0}} \Delta h_{k}(y) d y \\
= & \int_{B_{0}}(G(p, x)-G(x, y)) \Delta h_{k}(y) d y-\int_{B_{p}(R) \backslash B_{0}} G(x, y) \Delta h_{k}(y) d y \\
& \quad+\int_{\partial B_{p}(R)}\left(G(x, y) \frac{\partial h_{k}}{\partial \nu_{y}}-h_{k} \frac{\partial}{\partial \nu_{y}} G(x, y)\right)
\end{aligned}
$$

Clearly, the upper bound of $G$ implies that

$$
\limsup _{k \rightarrow \infty}\left|\int_{\partial B_{p}(R)}\left(G(x, y) \frac{\partial h_{k}}{\partial \nu_{y}}-h_{k} \frac{\partial}{\partial \nu_{y}} G(x, y)\right)\right| \leq C(n) R^{2-n}
$$

Also (0.3) and (0.5) imply that

$$
|G(p, x)-G(x, y)| \leq C(n) R_{0}\left(r-R_{0}\right)^{1-n},
$$

hence we conclude that

$$
\begin{aligned}
& \left|\limsup _{k \rightarrow \infty} \int_{B_{p}\left(R_{0}\right)}(G(p, x)-G(x, y)) \Delta h_{k}(y) d y\right| \\
& \leq \limsup _{k \rightarrow \infty}\left(\int_{B_{p}\left(R_{0}\right)}|\nabla G(x, y)|\left|\nabla h_{k}(y)\right|+\right. \\
& \left.\qquad \int_{\partial B_{p}\left(R_{0}\right)}|G(p, x)-G(x, y)|\left|\frac{\partial h_{k}(y)}{\partial \nu}\right|\right) \\
& =\int_{B_{p}\left(R_{0}\right)}|\nabla G(x, y)||\nabla h(y)|+\int_{\partial B_{p}\left(R_{0}\right)}|G(p, x)-G(x, y)|\left|\frac{\partial h(y)}{\partial \nu_{y}}\right| \\
& \leq C\left(R_{0}\right)\left(r-R_{0}\right)^{1-n} .
\end{aligned}
$$

For any $1>\epsilon>0$, noting that $\left|\nabla h_{k}\right|$ are uniformly bounded, and $\left|\nabla_{y} G\right|$ is 
integrable in $B_{x}(\epsilon r)$, we have

$$
\begin{aligned}
\limsup _{k \rightarrow \infty} & \left|\int_{B_{x}(\epsilon r)} G(x, y) \Delta h_{k} d y\right| \\
& \leq \limsup _{k \rightarrow \infty}\left|\int_{B_{x}(\epsilon r)}<\nabla_{y} G, \nabla h_{k}>\right|+\limsup _{k \rightarrow \infty}\left|\int_{\partial B_{x}(\epsilon r)} G \frac{\partial h_{k}}{\partial \nu_{y}}\right| \\
& =\left|\int_{B_{x}(\epsilon r)}<\nabla_{y} G, \nabla h>\right|+\left|\int_{\partial B_{x}(\epsilon r)} G \frac{\partial h}{\partial \nu_{y}}\right| \\
& \leq C(n)\left(r^{1-n} \int_{0}^{\epsilon r} \rho^{1-n} A_{x}(\rho) d \rho+\epsilon r^{2-n}\right) \\
& \leq \epsilon C(n) r^{2-n} .
\end{aligned}
$$

On the other hand, (1.1) together with $\Delta h_{k}(y) \geq-C d_{p}^{1-n}(y)$ on $B_{p}(R) \backslash B_{0}$ and the negative part $\left(\Delta h_{k}(y)\right)_{-}=\max \left\{-\Delta h_{k}(y), 0\right\}$ of $\Delta h_{k}(y)$ converges to zero almost everywhere, we conclude from (1.5) that

$$
\begin{aligned}
& \left|\limsup _{k \rightarrow \infty} \int_{B_{p}(R) \backslash B_{p}\left(R_{0}\right)} G(x, y) \Delta h_{k}(y) d y\right| \\
& \leq \limsup _{k \rightarrow \infty} \int_{B_{p}(R) \backslash\left(B_{p}\left(R_{0}\right) \cup B_{x}(\epsilon r)\right)} G(x, y)\left|\Delta h_{k}(y)\right| d y \\
& \quad+\limsup _{k \rightarrow \infty}\left|\int_{B_{x}(\epsilon r)} G(x, y) \Delta h_{k}(y) d y\right| \\
& \leq \limsup _{k \rightarrow \infty} C_{2}(\epsilon r)^{2-n} \int_{B_{p}(R) \backslash B_{p}\left(R_{0}\right)}\left|\Delta h_{k}(y)\right| d y+\epsilon C(n) r^{2-n} \\
& =\limsup _{k \rightarrow \infty} C_{2}(\epsilon r)^{2-n} \int_{B_{p}(R) \backslash B_{p}\left(R_{0}\right)} \Delta h_{k}(y) d y \\
& \quad+\limsup _{k \rightarrow \infty} 2 C_{2}(\epsilon r)^{2-n} \int_{B_{p}(R) \backslash B_{p}\left(R_{0}\right)}\left(\Delta h_{k}(y)\right)_{-} d y+\epsilon C(n) r^{2-n} \\
& =\limsup _{k \rightarrow \infty} C_{2}(\epsilon r)^{2-n}\left(\int_{\partial B_{p}(R)} \frac{\partial}{\partial \nu}\left(h_{k}(y)\right) d y-\int_{\partial B_{0}} \frac{\partial}{\partial \nu}\left(h_{k}(y)\right) d y\right) \\
& \quad+\epsilon C(n) r^{2-n} \\
& =C_{2}(\epsilon r)^{2-n} n(n-2)\left(\theta_{p}\left(R_{0}\right)-\theta_{p}(R)\right)+\epsilon C(n) r^{2-n} .
\end{aligned}
$$

Hence, combining (1.1), (1.3), (1.4) and (1.6), we conclude from (1.2) that

$$
\begin{aligned}
\mid h(x) & -n(n-2) \theta_{p}\left(R_{0}\right) G(p, x) \mid \\
& \leq C R^{2-n}+C(\epsilon r)^{2-n}\left(\theta_{p}\left(R_{0}\right)-\theta_{p}(R)\right)+C \epsilon r^{2-n}+C\left(R_{0}\right)\left(r-R_{0}\right)^{1-n} .
\end{aligned}
$$


Letting $R \rightarrow \infty$ and choosing $\epsilon=\eta_{p}^{\frac{1}{n-1}}\left(R_{0}\right)$, where $\eta_{p}\left(R_{0}\right)=\theta_{p}\left(R_{0}\right)-\theta$, this becomes

$$
\left|h(x)-n(n-2) \theta_{p}\left(R_{0}\right) G(p, x)\right| \leq C\left(C\left(R_{0}\right)\left(r-R_{0}\right)^{1-n}+\eta_{p}^{\frac{1}{n-1}}\left(R_{0}\right) r^{2-n}\right) .
$$

Multiplying both sides by $r^{n-2}=d_{p}^{n-2}(x)$, and letting $r \rightarrow \infty$, we have

$$
\limsup _{x \rightarrow \infty}\left|1-n(n-2) \theta_{p}\left(R_{0}\right) d^{n-2}(p, x) G(p, x)\right| \leq C(n, \theta) \eta_{p}^{\frac{1}{n-1}}\left(R_{0}\right)
$$

The theorem now follows by letting $R_{0} \rightarrow \infty$.

We should remark that when $n=2$, any manifold with non-negative curvature is parabolic. Hence any Green's function must change sign. However, if $M^{2}$ has non-negative curvature and has maximal volume growth, then $M$ must be conformally equivalent to $\mathbb{R}^{2}$. In particular, we can write the metric on $M$ as $d s^{2}=e^{2 u} d s_{0}^{2}$ where $d s_{0}^{2}$ is the Euclidean metric on $\mathbb{R}^{2}$ and $u$ is a smooth function. Let us denote the Euclidean distant between any two points $p, x \in M$ by $d_{0}(p, x)$. Using the fact that the Laplacian is conformally invariant in dimension 2, we see that the Euclidean Green's function $G(p, x)=\frac{1}{2 \pi} \log d_{0}(p, x)$ is also a Green's function with respect to the metric $d s^{2}$. It was shown in [L-T 1] (Corollary 3.3) that

$$
\lim _{x \rightarrow \infty} \frac{\log d(p, x)}{\log d_{0}(p, x)}=\frac{\theta}{\pi}
$$

Hence, in this case, we also have the asymptotic behavior

$$
\lim _{x \rightarrow \infty} \frac{2 \theta G(p, x)}{\log d(p, x)}=1 .
$$

In fact, in [L-T 1] the author considered the larger class of surfaces, namely, surfaces with finite total curvature. Estimates similar to the one stated above holds for surfaces with finite total curvature and quadratic volume growth. In our next theorem, we prove a slightly stronger result than Theorem 0.3 in $[\mathrm{C}-\mathrm{M}]$.

Theorem 1.2. Let $M^{n}$ be a complete noncompact manifold with non-negative Ricci curvature. Assume that $n \geq 3$ and let $p \in M$ be a fixed point. Suppose $M$ admits a positive Green's function $G(x, y)$ and let $u$ be any positive harmonic function defined on $M \backslash B_{p}(1)$. Then there exist constants $a, b$, and $C>0$, and a function $v$ which is harmonic on $M \backslash B_{p}(3 / 2)$ satisfying

$$
|v(x)| \leq C d^{-1}(p, x) G(p, x),
$$

such that,

$$
u(x)=a+b G(p, x)+v(x)
$$


on $M \backslash B_{p}(2)$. In fact, the constant $b$ is given by $b=-\int_{\partial B_{p}(2)} \frac{\partial u}{\partial r}$.

Proof. Note that an argument of Milnor [M] asserts that $M$ has finite first Betti number. The assumption that $M$ is non-parabolic ([L-Y] and $[\mathrm{C}-\mathrm{G}])$ implies that $M$ has only one end. It was proved in [L-T 2] that the $\operatorname{limit}_{x \rightarrow \infty} u(x)=a$ exists, and must be finite. Let us first extend $u$ to a smooth function on $M$ such that $u$ remains unchanged outside $B_{p}(3 / 2)$. Define $h=\Delta u$ which has support in $B_{p}(3 / 2)$ and

$$
u(x)=a-\int_{M} G(x, y) h(y) d y .
$$

In particular, for $x \neq p$,

$$
\begin{aligned}
u(x) & =a-\int_{B_{p}(3 / 2)} G(x, y) h(y) d y \\
& =a-G(p, x) \int_{B_{p}(3 / 2)} h(y) d y+\int_{B_{p}(3 / 2)}(G(p, x)-G(x, y)) h(y) d y \\
& =a+b G(p, x)+v(x),
\end{aligned}
$$

where $b=-\int_{B_{p}(3 / 2)} h(y) d y$, and $v(x)=\int_{B_{p}(3 / 2)}(G(p, x)-G(x, y)) h(y) d y$. One checks readily that $v(x)$ is harmonic on $M \backslash B_{p}(3 / 2)$. As in the proof of the previous theorem, one can show that there exists $C>0$, such that,

$$
|v(x)| \leq C d^{-1}(p, x) G(p, x)
$$

for $x \notin B_{p}(2)$. This completes the proof of the theorem.

\section{$\S 2$ Heat kernel estimates}

In this section, we will continue to assume that $M$ has non-negative Ricci curvature and maximal volume growth. The goal of the next theorem is to prove sharp pointwise upper and lower bounds for the heat kernel on such a manifold. The heat kernel estimates are valid for all dimensions. When $n \geq 3$, after integrating with respect to time, we obtain sharp pointwise estimate for the Green's function. Moreover, if we let $d(p, q) \rightarrow \infty$, then these upper and lower bounds will reduce to the asymptotic estimates proved in Theorem 1.1.

Theorem 2.1. Let $M$ be a complete manifold with non-negative Ricci curvature and maximal volume growth. For any $\delta>0$, the heat kernel of $M$ must satisfy the estimate

$$
\begin{aligned}
\frac{\omega_{n}}{\theta_{p}(\delta d(p, q))} & (4 \pi t)^{-\frac{n}{2}} \exp \left(-\frac{1+9 \delta}{4 t} d^{2}(p, q)\right) \\
& \leq H(p, q, t) \\
& \leq\left(1+C_{14}(\delta+\beta)\right) \frac{\omega_{n}}{\theta}(4 \pi t)^{-\frac{n}{2}} \exp \left(-\frac{1-\delta}{4 t} d^{2}(p, q)\right)
\end{aligned}
$$


where $C_{14}>0$ is a constant depending only on $n$ and $\theta$, and $\beta$ is given by

$$
\beta=\delta^{-2 n} \max _{r \geq(1-\delta) d(p, q)}\left\{1-\frac{\theta_{p}(r)}{\theta_{p}\left(\delta^{2 n+1} r\right)}\right\}
$$

Proof. Using the Cheeger-Yau [C-Y] comparison theorem, Li-Yau [L-Y] showed that for any $R>0$,

$$
\int_{B_{p}(R)} H(x, q, t) d x \geq \int_{B_{\bar{p}}(R)} \bar{H}(\bar{x}, \bar{q}, t) d \bar{x}
$$

where $\bar{H}$ is the heat kernel on $\mathbb{R}^{n}$ and $d(p, q)=d(\bar{p}, \bar{q})$. On the other hand, the Harnack inequality of $[\mathrm{L}-\mathrm{Y}]$ implies that for $\eta>0$,

$$
H(x, q, t) \leq(1+\eta)^{\frac{n}{2}} \exp \left(\frac{d^{2}(x, p)}{4 \eta t}\right) H(p, q,(1+\eta) t)
$$

Hence, applying this to both $H$ and $\bar{H}$, we have

$$
\begin{aligned}
& H\left(p, q,(1+\eta)^{2} t\right) \\
& \quad \geq V_{p}(R)^{-1}(1+\eta)^{-\frac{n}{2}} \exp \left(\frac{-R^{2}}{4 \eta(1+\eta) t}\right) \int_{B_{\bar{p}}(R)} \bar{H}(\bar{x}, \bar{q},(1+\eta) t) d \bar{x} \\
& \quad \geq \omega_{n} R^{n} V_{p}(R)^{-1}(1+\eta)^{-n} \exp \left(\frac{-(2+\eta) R^{2}}{4 \eta(1+\eta) t}\right) \bar{H}(\bar{p}, \bar{q}, t) .
\end{aligned}
$$

Setting $R=\eta d(p, q)$ and writing $V_{p}(R)=\theta_{p}(R) R^{n}$, we have

$H\left(p, q,(1+\eta)^{2} t\right) \geq \frac{\omega_{n}}{\theta_{p}(\eta d(p, q))}(1+\eta)^{-n} \exp \left(\frac{-\eta(2+\eta) d^{2}(p, q)}{4(1+\eta) t}\right) \bar{H}(\bar{p}, \bar{q}, t)$

Rewriting this and letting $s=(1+\eta)^{2} t$, we conclude that for any $\eta>0$,

$$
H(p, q, s) \geq \frac{\omega_{n}}{\theta_{p}(\eta d(p, q))}(4 \pi s)^{-\frac{n}{2}} \exp \left(-\frac{(1+9 \eta) d^{2}(p, q)}{4 s}\right)
$$

which is the desired lower estimate. 
To prove the upper bound, let us first recall that it was proved in [L] that for a fixed point $p \in M$, the function $t^{\frac{n}{2}} H(p, p, t)$ is monotonically non-decreasing with

$$
t^{\frac{n}{2}} H(p, p, t) \nearrow \frac{\omega_{n}}{(4 \pi)^{n / 2} \theta}
$$

Let $\delta>0$ be sufficiently small, and for $d(p, q) \leq \delta \sqrt{t},(2.2)$ implies that

$$
\begin{aligned}
H(p, q, t) & \leq(1+\delta)^{n / 2} \exp \left(\frac{d^{2}(p, q)}{4 \delta t}\right) H(p, p,(1+\delta) t) \\
& \leq(4 \pi t)^{-n / 2} \frac{\omega_{n}}{\theta} \exp \left(\frac{d^{2}(p, q)}{4 \delta t}\right) \\
& \leq(4 \pi t)^{-n / 2} \frac{\omega_{n}}{\theta} \exp \left(-\frac{d^{2}(p, q)}{4 t}\right) \exp \left(\frac{\delta^{2}+\delta}{4}\right) \\
& \leq\left(1+C_{15} \delta\right)(4 \pi t)^{-n / 2} \frac{\omega_{n}}{\theta} \exp \left(-\frac{d^{2}(p, q)}{4 t}\right)
\end{aligned}
$$

where $C_{15}>0$ is some positive constant. Let us now consider the case $d(p, q) \geq$ $\delta \sqrt{t}$, and let $R=(1-\delta) d(p, q)$. Since

$$
\int_{M} H(p, x, s) d x=\int_{\mathbb{R}^{n}} \bar{H}(\bar{p}, \bar{x}, s) d \bar{x}=1
$$

inequality (2.1) implies

$$
\int_{M \backslash B_{p}(R)} H(p, x,(1+\delta) t) d x \leq \int_{\mathbb{R}^{n} \backslash B_{\bar{p}}(R)} \bar{H}(\bar{p}, \bar{x},(1+\delta) t) d \bar{x} .
$$

Using the fact that $B_{p}(R) \cap B_{q}(\delta R)=\emptyset$, we have

$$
\begin{aligned}
\int_{B_{q}(\delta R)} H & (p, x,(1+\delta) t) d x \\
\leq & \int_{\mathbb{R}^{n} \backslash B_{\bar{p}}(R)} \bar{H}(\bar{p}, \bar{x},(1+\delta) t) d \bar{x} \\
& -\int_{M \backslash\left(B_{p}(R) \cup B_{q}(\delta R)\right)} H(p, x,(1+\delta) t) d x .
\end{aligned}
$$


Invoking the lower bound $(2.3)$ of $H(p, q, s)$ and noting that $\theta_{p}(r) \searrow \theta$, we get

$$
\begin{aligned}
& \int_{B_{q}(\delta R)} H(p, x,(1+\delta) t) d x \\
& \leq \int_{\mathbb{R}^{n} \backslash B_{\bar{p}}(R)} \bar{H}(\bar{p}, \bar{x},(1+\delta) t) d \bar{x}-(4 \pi(1+\delta) t)^{-\frac{n}{2}} \\
& \times \int_{M \backslash\left(B_{p}(R) \cup B_{q}(\delta R)\right)} \frac{\omega_{n}}{\theta_{p}(\eta d(p, x))} \exp \left(-\frac{(1+9 \eta) d^{2}(p, x)}{4(1+\delta) t}\right) d x \\
& \leq n \omega_{n}(4 \pi(1+\delta) t)^{-\frac{n}{2}} \int_{R}^{\infty} r^{n-1} \exp \left(-\frac{r^{2}}{4(1+\delta) t}\right) d r \\
&-(4 \pi(1+\delta) t)^{-\frac{n}{2}} \int_{M \backslash B_{p}(R)} \frac{\omega_{n}}{\theta_{p}(\eta d(p, x))} \exp \left(-\frac{(1+9 \eta) d^{2}(p, x)}{4(1+\delta) t}\right) d x \\
&+(4 \pi(1+\delta) t)^{-\frac{n}{2}} \int_{B_{q}(\delta R)} \frac{\omega_{n}}{\theta_{p}(\eta d(p, x))} \exp \left(-\frac{(1+9 \eta) d^{2}(p, x)}{4(1+\delta) t}\right) d x \\
& \leq n \omega_{n}(4 \pi(1+\delta) t)^{-\frac{n}{2}} \int_{R}^{\infty} r^{n-1} \exp \left(-\frac{r^{2}}{4(1+\delta) t}\right) d r \\
&-n \omega_{n}(4 \pi(1+\delta) t)^{-\frac{n}{2}} \int_{R}^{\infty} \exp \left(-\frac{(1+9 \eta) r^{2}}{4(1+\delta) t}\right) \frac{A_{p}(r)}{n \theta_{p}(\eta r)} d r \\
&+\frac{\omega_{n}}{\theta}(4 \pi(1+\delta) t)^{-\frac{n}{2}} \exp \left(-\frac{1+9 \eta}{4(1+\delta) t}(d(p, q)-\delta R)^{2}\right) V_{q}(\delta R) .
\end{aligned}
$$

Rewriting the first two terms on the right hand side as

$$
\begin{aligned}
n \omega_{n}(4 & \pi(1+\delta) t)^{-\frac{n}{2}} \int_{R}^{\infty} r^{n-1}\left(\exp \left(-\frac{r^{2}}{4(1+\delta) t}\right)-\exp \left(-\frac{(1+9 \eta) r^{2}}{4(1+\delta) t}\right)\right) d r \\
& +n \omega_{n}(4 \pi(1+\delta) t)^{-\frac{n}{2}} \int_{R}^{\infty} r^{n-1} \exp \left(-\frac{(1+9 \eta) r^{2}}{4(1+\delta) t}\right)\left(1-\frac{\theta_{p}(r)}{\theta_{p}(\eta r)}\right) d r
\end{aligned}
$$

we have

$$
\begin{aligned}
& \int_{B_{q}(\delta R)} H(p, x,(1+\delta) t) d x \\
& \leq n \omega_{n}(4 \pi(1+\delta) t)^{-\frac{n}{2}} \int_{R}^{\infty} r^{n-1} \exp \left(-\frac{r^{2}}{4(1+\delta) t}\right) \frac{9 \eta r^{2}}{4(1+\delta) t} d r \\
&+ n \omega_{n}(4 \pi(1+\delta) t)^{-\frac{n}{2}} \max _{r \geq R}\left\{1-\frac{\theta_{p}(r)}{\theta_{p}(\eta r)}\right\} \\
& \quad \times \int_{R}^{\infty} r^{n-1} \exp \left(-\frac{(1+9 \eta) r^{2}}{4(1+\delta) t}\right) d r \\
&+\frac{\omega_{n}}{\theta}(4 \pi(1+\delta) t)^{-\frac{n}{2}} \exp \left(-\frac{1+9 \eta}{4(1+\delta) t}(d(p, q)-\delta R)^{2}\right) V_{q}(\delta R)
\end{aligned}
$$


Direct computation shows that

$$
\begin{aligned}
(4 \pi(1+\delta) t)^{-\frac{n}{2}} & \int_{R}^{\infty} r^{n-1} \exp \left(-\frac{r^{2}}{4(1+\delta) t}\right) \frac{r^{2}}{4(1+\delta) t} d r \\
& \leq C_{16}\left(1+R^{n}(4 \pi(1+\delta) t)^{-\frac{n}{2}}\right) \exp \left(-\frac{R^{2}}{4(1+\delta) t}\right)
\end{aligned}
$$

for some constant $C_{16}>0$ depending only on $n$. Also, there exists constants $C_{17}$ and $C_{18}$ depending on $n$, such that,

$$
\begin{aligned}
(4 \pi(1+\delta) t)^{-\frac{n}{2}} \int_{R}^{\infty} r^{n-1} \exp \left(-\frac{(1+9 \eta) r^{2}}{4(1+\delta) t}\right) d r \\
=(4 \pi(1+\delta) t)^{-\frac{n}{2}} \frac{1}{n}\left[r^{n} \exp \left(-\frac{(1+9 \eta) r^{2}}{4(1+\delta) t}\right)\right]_{R}^{\infty} \\
\quad+(4 \pi(1+\delta) t)^{-\frac{n}{2}} \frac{1}{n} \int_{R}^{\infty} r^{n} \exp \left(-\frac{(1+9 \eta) r^{2}}{4(1+\delta) t}\right) \frac{2(1+9 \eta) r}{4(1+\delta) t} d r \\
\leq C_{17}(4 \pi(1+\delta) t)^{-\frac{n}{2}} \int_{R}^{\infty} r^{n-1} \exp \left(-\frac{(1+9 \eta) r^{2}}{4(1+\delta) t}\right) \frac{r^{2}}{4(1+\delta) t} d r \\
\leq C_{18}\left(1+R^{n}(4 \pi(1+\delta) t)^{-\frac{n}{2}}\right) \exp \left(-\frac{R^{2}}{4(1+\delta) t}\right)
\end{aligned}
$$

Combining (2.6) and (2.7) with (2.5), we conclude that

$$
\begin{aligned}
& \int_{B_{q}(\delta R)} H(p, x,(1+\delta) t) d x \\
& \leq \frac{\omega_{n}}{\theta}(4 \pi(1+\delta) t)^{-\frac{n}{2}} \exp \left(-\frac{1+9 \eta}{4(1+\delta) t}(d(p, q)-\delta R)^{2}\right) V_{q}(\delta R) \\
& \quad+C_{18}\left(1+R^{n}(4 \pi(1+\delta) t)^{-\frac{n}{2}}\right)(\eta+\alpha(\eta, R)) \exp \left(-\frac{R^{2}}{4(1+\delta) t}\right)
\end{aligned}
$$

where

$$
\alpha(\eta, R)=\max _{r \geq R}\left\{1-\frac{\theta_{p}(r)}{\theta_{p}(\eta r)}\right\} .
$$


Note that $\theta_{p}(R) \searrow \theta$ implies $\alpha(\eta, R) \rightarrow 0$. Using (2.2) and (2.8), we obtain

$$
\begin{aligned}
& H(p, q, t) \\
& \leq(1+\delta)^{\frac{n}{2}} \exp \left(\frac{\delta^{2} R^{2}}{4 \delta t}\right) V_{q}^{-1}(\delta R) \int_{B_{q}(\delta R)} H(p, x,(1+\delta) t) d x \\
& \leq \frac{\omega_{n}}{\theta}(1+\delta)^{\frac{n}{2}} \exp \left(\frac{\delta R^{2}}{4 t}\right)(4 \pi(1+\delta) t)^{-\frac{n}{2}} \\
& \quad \exp \left(-\frac{1+9 \eta}{4(1+\delta) t}(d(p, q)-\delta R)^{2}\right) \\
& \quad+C_{19}(\eta+\alpha(\eta, R)) \exp \left(-\frac{R^{2}}{4(1+\delta) t}\right) \\
& \quad \times\left(V_{q}^{-1}(\delta R)+\frac{R^{n}}{V_{q}(\delta R)}(4 \pi(1+\delta) t)^{-\frac{n}{2}}\right) .
\end{aligned}
$$

Notice that $V_{q}(\delta R) \geq \theta(\delta R)^{n}$ and also

$$
R=(1-\delta) d(p, q) \geq \delta(1-\delta) \sqrt{t}
$$

Therefore, we conclude from (2.9) that

$$
\begin{aligned}
& H(p, q, t) \\
& \quad \leq \frac{\omega_{n}}{\theta}(4 \pi t)^{-\frac{n}{2}} \exp \left(\left(-\frac{(1+9 \eta)(1-\delta)^{2}}{4(1+\delta) t}+\frac{\delta(1-\delta)^{2}}{4 t}\right) d^{2}(p, q)\right) \\
& \quad+C_{14}(\eta+\alpha(\eta, R)) \delta^{-2 n}(4 \pi t)^{-\frac{n}{2}} \exp \left(-\frac{(1-\delta)^{2}}{4(1+\delta) t} d^{2}(p, q)\right) .
\end{aligned}
$$

Choosing $\eta=\delta^{2 n+1}$ and writing

$$
\beta=\delta^{-2 n} \alpha(\eta,(1-\delta) d(p, q)),
$$

we derive from (2.10) that

$$
H(p, q, t) \leq\left(1+C_{14}(\delta+\beta)\right) \frac{\omega_{n}}{\theta}(4 \pi t)^{-\frac{n}{2}} \exp \left(-\frac{1-\delta}{4 t} d^{2}(p, q)\right) .
$$

Combining (2.4) and (2.11), we obtain the desired upper estimate.

Corollary 2.2. Let $M$ satisfy the same assumption as Theorem 2.1, and $n \geq 3$.

Then the minimal positive Green's function must satisfy

$$
\begin{aligned}
& (1+9 \delta)^{1-\frac{n}{2}} \frac{d^{2-n}(p, q)}{n(n-2) \theta_{p}(\delta d(p, q))} \\
& \quad \leq G(p, q) \\
& \quad \leq\left(1+C_{14}(\delta+\beta)\right)(1-\delta)^{1-\frac{n}{2}} \frac{d^{2-n}(p, q)}{n(n-2) \theta} .
\end{aligned}
$$


Proof. Let us first observe that

$$
\begin{aligned}
\int_{0}^{\infty}(4 \pi t)^{-\frac{n}{2}} \exp \left(\frac{-\gamma d^{2}(p, q)}{4 t}\right) d t & =\gamma^{1-\frac{n}{2}} \int_{0}^{\infty}(4 \pi s)^{-\frac{n}{2}} \exp \left(\frac{-d^{2}(p, q)}{4 s}\right) d s \\
& =\frac{1}{n(n-2) \omega_{n}} \gamma^{1-\frac{n}{2}} d^{2-n}(p, q) .
\end{aligned}
$$

Integrating over $0<t<\infty$ and using the lower bound of $H(p, q, t)$, we have

$$
\begin{aligned}
G(p, q) & =\int_{0}^{\infty} H(p, q, t) d t \\
& \geq \frac{\omega_{n}}{\theta_{p}(\delta d(p, q))} \int_{0}^{\infty}(4 \pi t)^{-\frac{n}{2}} \exp \left(\frac{-(1+9 \delta) d^{2}(p, q)}{4 t}\right) d t \\
& =\frac{1}{n(n-2) \theta_{p}(\delta d(p, q))}(1+9 \delta)^{1-\frac{n}{2}} d^{2-n}(p, q) .
\end{aligned}
$$

Similarly, if we use the upper bound estimate of $H(p, q, t)$, then

$$
\begin{aligned}
G(p, q)= & \int_{0}^{\infty} H(p, q, t) d t \\
\leq & \left(1+C_{14}(\delta+\beta)\right) \frac{\omega_{n}}{\theta} \\
& \times \int_{0}^{\infty}(4 \pi t)^{-\frac{n}{2}} \exp \left(\frac{-(1-\delta) d^{2}(p, q)}{4 t}\right) d t \\
= & \left(1+C_{14}(\delta+\beta)\right) \frac{1}{n(n-2) \theta}(1-\delta)^{1-\frac{n}{2}} d^{2-n}(p, q) .
\end{aligned}
$$

Note that by letting $q \rightarrow \infty$, and using the fact that $\theta_{p}(\delta d(p, q)) \rightarrow \theta$ we conclude that

$$
\lim _{q \rightarrow \infty} d^{n-2}(p, q) G(p, q) \geq \frac{1}{n(n-2) \theta}(1+9 \delta)^{1-\frac{n}{2}}
$$

The sharp asymptotic lower bound follows by letting $\delta \rightarrow 0$. Similarly, the sharp asymptotic upper bound also follows by using the fact that $\beta \rightarrow 0$ and then $\delta \rightarrow 0$.

The following corollary gives a slightly stronger result concerning the asymptotic behavior in the time variable for the heat kernel as was proved in $[\mathrm{L}]$.

Corollary 2.3. Let $M$ be as in Theorem 2.1. If $\gamma(t)=(q(t), t)$ is any path on $M \times(0, \infty)$ satisfying $d^{2}(q(t), p)=O(t)$ as $t \rightarrow \infty$, then

$$
\lim _{t \rightarrow \infty} V_{p}(\sqrt{t}) \exp \left(\frac{d^{2}(p, q(t))}{4 t}\right) H(p, q(t), t)=\omega_{n}(4 \pi)^{-\frac{n}{2}} .
$$


Proof. If $d^{2}(q(t), p)=o(t)$ as $t \rightarrow \infty$, then the result follows from [L]. Without loss of generality, we may assume that $d(q(t), p) \rightarrow \infty$ as $t \rightarrow \infty$. In particular, $\theta_{p}(\delta d(p, q(t))) \rightarrow \theta$ and $\beta \rightarrow 0$ as $t \rightarrow \infty$. Applying the heat kernel bounds of Theorem 2.1 and letting $t \rightarrow \infty$, we have

$$
\begin{aligned}
\liminf _{t \rightarrow \infty} & V_{p}(\sqrt{t}) \exp \left(\frac{d^{2}(p, q(t))}{4 t}\right) H(p, q(t), t) \\
& \geq \omega_{n}(4 \pi)^{-\frac{n}{2}} \lim _{t \rightarrow \infty} \frac{V_{p}(\sqrt{t})}{\theta(\sqrt{t})^{n}} \exp \left(-\frac{9 \delta d^{2}(p, q(t))}{4 t}\right) \\
& \geq \omega_{n}(4 \pi)^{-\frac{n}{2}} \exp \left(-C_{20} \delta\right)
\end{aligned}
$$

for some constant $C_{20}>0$. Letting $\delta \rightarrow 0$, we conclude that

$$
\liminf _{t \rightarrow \infty} V_{p}(\sqrt{t}) \exp \left(\frac{d^{2}(p, q(t))}{4 t}\right) H(p, q(t), t) \geq \omega_{n}(4 \pi)^{-\frac{n}{2}}
$$

Similarly, we have

$$
\begin{aligned}
\limsup _{t \rightarrow \infty} & V_{p}(\sqrt{t}) \exp \left(\frac{d^{2}(p, q(t))}{4 t}\right) H(p, q(t), t) \\
\leq & \left(1+C_{14}(\delta+\beta)\right) \omega_{n}(4 \pi)^{-\frac{n}{2}} \lim _{t \rightarrow \infty} \frac{V_{p}(\sqrt{t})}{\theta(\sqrt{t})^{n}} \exp \left(\frac{\delta d^{2}(p, q(t))}{4 t}\right) \\
\leq & \omega_{n}(4 \pi)^{-\frac{n}{2}} \exp \left(C_{21} \delta\right),
\end{aligned}
$$

where $C_{21}>0$ is a constant. Thus, by taking $\delta \rightarrow 0$, we have

$$
\limsup _{t \rightarrow \infty} V_{p}(\sqrt{t}) \exp \left(\frac{d^{2}(p, q(t))}{4 t}\right) H(p, q(t), t) \leq \omega_{n}(4 \pi)^{-\frac{n}{2}}
$$

and the corollary follows.

\section{Acknowledgment}

The authors would like to thank Gang Tian and Shing Tung Yau for their interest in this work. Part of this work was done when the first and the third author were visiting MIT. They would like to express their gratitude to Gang Tian and the Mathematics Department of MIT for their hospitality.

\section{References}

[B-C] R. Bishop and R. Crittenden, Geometry of manifolds, Academic Press, New York, 1964.

[C-G] J. Cheeger and D. Gromoll, The splitting theorem for manifolds of nonnegative Ricci curvature, J. Diff. Geom. 6 (1971), 119-128. 
[C-Y] J. Cheeger and S. T. Yau, A lower bound for the heat kernel, Comm. Pure Appl. Math. 34 (1981), 465-480.

[Cg-Y] S. Y. Cheng and S. T. Yau, Differential equations on Riemannian manifolds and their geometric applications, Comm. Pure Appl. Math. 28 (1975), 333-354.

[C] T. H. Colding, Ricci curvature and volume convergence, preprint.

[C-M] T. H. Colding and W. P. Minicozzi II, Large scale behavior of kernels of Schrödinger operators, preprint.

[G-W] R. Greene and H. Wu, Function theory on manifolds which possess a pole, SpringerVerlag Lecture Notes in Mathematics, 699, Berlin, 1979.

[L] P. Li, Large time behavior of the heat equation on complete manifolds with nonnegative Ricci curvature, Ann. of Math. 124 (1986), 1-21.

[L-T 1] P. Li and L. F. Tam, Complete surfaces with finite total curvature, J. Diff. Geom. 33 (1991), 139-168.

[L-T 2] , Green's functions, harmonic functions and volume comparison, J. Diff. Geom. 41 (1995), 277-318.

[L-Y] P. Li and S. T. Yau, On the parabolic kernel of the Schrödinger operator, Acta Math. 156 (1986), 153-201.

[M] J. Milnor, A note on curvature and fundamental group, J. Diff. Geom. 2 (1968), 1-7.

[V] N. Varopoulos, The Poisson kernel on positively curved manifolds, J. Funct. Anal. 44 (1981), 359-380.

Department of Mathematics, University of California, Irvine, Irvine, CA 926973875

E-mail address: pli@math.uci.edu

Department of Mathematics, Chinese University of Hong Kong, Shatin, NT, Hong KONG

E-mail address: lftam@math.cuhk.hk,

Department of Mathematics, Massachusetts Institute of Technology, Cambridge, MA 02139-4307

E-mail address: jwang@math.mit.edu 